\title{
A brief overview of the research reactor FRM II
}

\author{
H. Gerstenberg, I. Neuhaus \\ Forschungsneutronenquelle Heinz Maier-Leibnitz (FRM II), TU München, Lichtenbergstrasse 1, \\ 85748 Garching, Germany
}

heiko.gerstenberg@frm2.tum.de

\begin{abstract}
The design of the Forschungsneutronenquelle Heinz Maier-Leibnitz (FRM II) is characterized by a single compact fuel element being embedded in a large heavy water filled moderator tank. In this geometry the peak value of the thermal neutron flux density is located outside the fuel element in an area which is accessible for major experimental installations like the Cold Neutron Source. A further advantage of the use of heavy water is that it results in a very high ratio of thermal/fast neutron flux density offering ideal conditions for most irradiation experiments. Consequently FRM II was equipped with a number of irradiation facilities covering a large scope of applications.

In order to attract not only scientists but also commercial customers FRM II operates a semi-automatic Si doping facility for ingots up to $200 \mathrm{~mm}$ in diameter and $500 \mathrm{~mm}$ in height and a wide range of target resistivities between $20 \Omega \mathrm{cm}$ and $1100 \Omega \mathrm{cm}$. Due to the still growing demand for neutron transmutation doped silicon a total amount of approximately $4000 \mathrm{~kg}$ Si was irradiated already in the first year of operation. Another application which merits special attention is the therapy of mainly skin and throat cancer by means of fast neutron irradiation. Starting in 2007 the first human patients were treated in FRM II. The available irradiation service at FRM II is completed by standard experimental tools like a pneumatic and a hydraulic rabbit system, being used by numerous users with scientific and industrial background as well.
\end{abstract}

\section{Keywords}

Reactor design, neutron irradiation, Si doping

\section{INTRODUCTION}

Since 2005 the Technische Universität München (TUM) is operating on its campus in Garching near Munich Germany's most modern research reactor, the Forschungsneutronenquelle Heinz Maier-Leibnitz (FRM II). In the previous years it was built and commissioned in collaboration between the TUM and the Siemens/AREVA company. The results of the commissioning being the basis of the operation license were reported earlier (Schreckenbach 2005).

Although the FRM II was clearly designed and optimized for the production of neutrons for the basic research by means of beam tube experiments there was a general agreement already since the early design phase that the reactor will also be made available to applied science and industrial applications. Consequently its basic equipment did not only provide 12 beam tubes and secondary sources like a Cold Neutron Source and a Hot Neutron Source but also a number of irradiation facilities which cover a large variety of experimental needs in topics as different as neutron activation analysis, silicon doping or cancer treatment by irradiation with fast neutrons.

\section{GENERAL REACTOR DESIGN}

The FRM II is a heavy water moderated, light water cooled research reactor exhibiting a thermal power of $20 \mathrm{MW}$. Its central component is the single cylindrical fuel element (see figure 1) which forms the reactor core. The fuel in use is $\mathrm{U}_{3} \mathrm{Si}_{2}$ dispersed in Al. Each fuel element contains approximately $8.1 \mathrm{~kg}$ of uranium enriched to $92.5 \%$ in U-235. In more detail the fuel element contains 113 separate fuel plates which are mounted between an inner and an outer tube with diameters of $\emptyset_{\mathrm{i}}=118 \mathrm{~mm}$ and $\varnothing_{\mathrm{o}}=237 \mathrm{~mm}$ respectively. The Hf-control rod is moved within the inner tube of the fuel element. The fuel plates are bent to an involute shape in order to guarantee a constant width of the cooling slits of $2.3 \mathrm{~mm}$. Because of the compactness of the fuel element the moderation of the neutrons takes place in the surrounding heavy water tank, an effect that made it necessary to decrease the fuel density in the outer part of the plates from 3 $\mathrm{g} / \mathrm{cm}^{3}$ to $1.5 \mathrm{~g} / \mathrm{cm}^{3}$ in order to avoid an unacceptable power peak at the outer surface of the fuel element. The cooling is established by light water being pumped through the cooling slits at a rate of $300 \mathrm{~kg} / \mathrm{s}$ and leading to a temperature increase of the cooling water of only about $15^{\circ} \mathrm{C}$, i.e. from $\sim 35^{\circ} \mathrm{C}$ to $\sim 50^{\circ} \mathrm{C}$. The cooling stream is provided by four primary pumps and the thermal power of $20 \mathrm{MW}$ is released through a total of three cooling circuits to the air.

Originally the lifetime of a fuel element was 52 full power days. Fortunately, however, the irradiation experiments for the certification of the fuel which had been conducted during the project phase of FRM II covered already a higher burn-up of the fuel. 


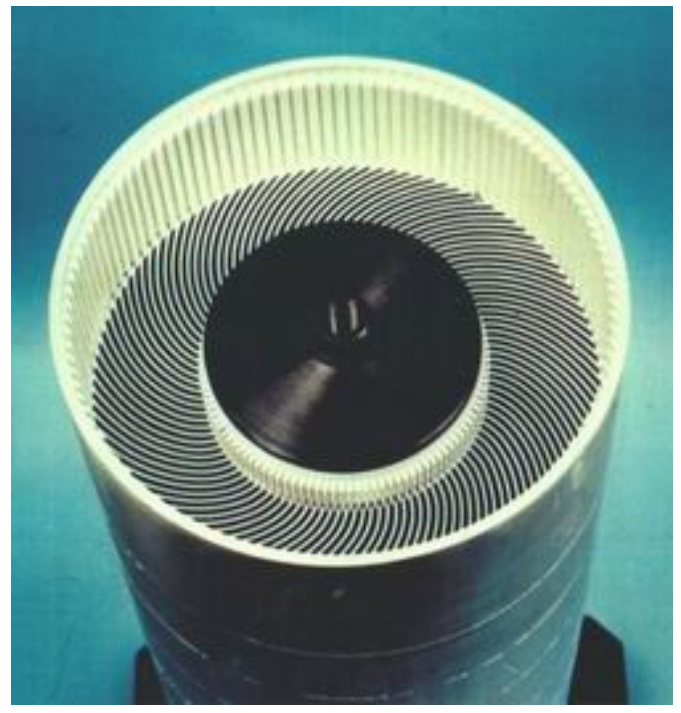

Figure 1: Top view to the FRM II fuel element

Therefore, taking into account the experience from the first 12 reactor cycles and after careful evaluation of important operational parameters like e.g. the sensitivity of the control rod at the end of the rector cycle and the low effect of swelling of the fuel plates with increasing burn-up the TUM applied for the agreement to extend the maximum cycle length to 60 full power days which was awarded by the local German authorities in December 2007. Due to the high cost of fuel, fabrication of fuel elements, transportation, and disposal of irradiated fuel the extension of the cycle length provides a considerable economical benefit. The maximum number of yearly days of reactor operation, on the other hand, was kept constant at 260 days which turns out to be necessary because of the high amount of obligatory maintenance and periodic testing, a big portion of which has to be done in the presence of external, independent experts. It is noteworthy, however, that already in 2006, the second year of reactor operation, the goal of 260 full power days was achieved for the first time. As mentioned above the fuel element is surrounded by the heavy water containing moderator tank which is also cylindrical in shape with diameter and height of equally approximately $2.5 \mathrm{~m}$. Strictly speaking the fuel element is contained in the central channel of the moderator tank which is part of the barrier between the light and heavy water circuits of FRM II. Besides five shut down rods the moderator tank contains the thimbles of the beam tubes, the secondary sources and the irradiation channels of all of the irradiation devices for material sciences. Figure 2 gives an overview of the conceptual design of the FRM II.

\section{IRRADIATION SERVICE AT THE FRM II}

The different irradiation facilities of the FRM II will be described in some detail below. For all irradiation facilities, however, there are some requirements to be met before an irradiation is accepted for being performed. Maybe the most important one is an in advance calculation of the estimated activity and dose rate of the sample to be irradiated and the compliance of the result with the limiting values stated in the license for the nuclear operation of the FRM II. In addition for external customers it is evident to posses a handling license for unsealed radioactive sources. Finally the application form for irradiations is checked from the head of the irradiation division and the responsible person for radiation protection with respect to possible problems arising from sample heating, radiolysis or non-suitable packaging. Not before all these aspects have been evaluated the clearance is given.

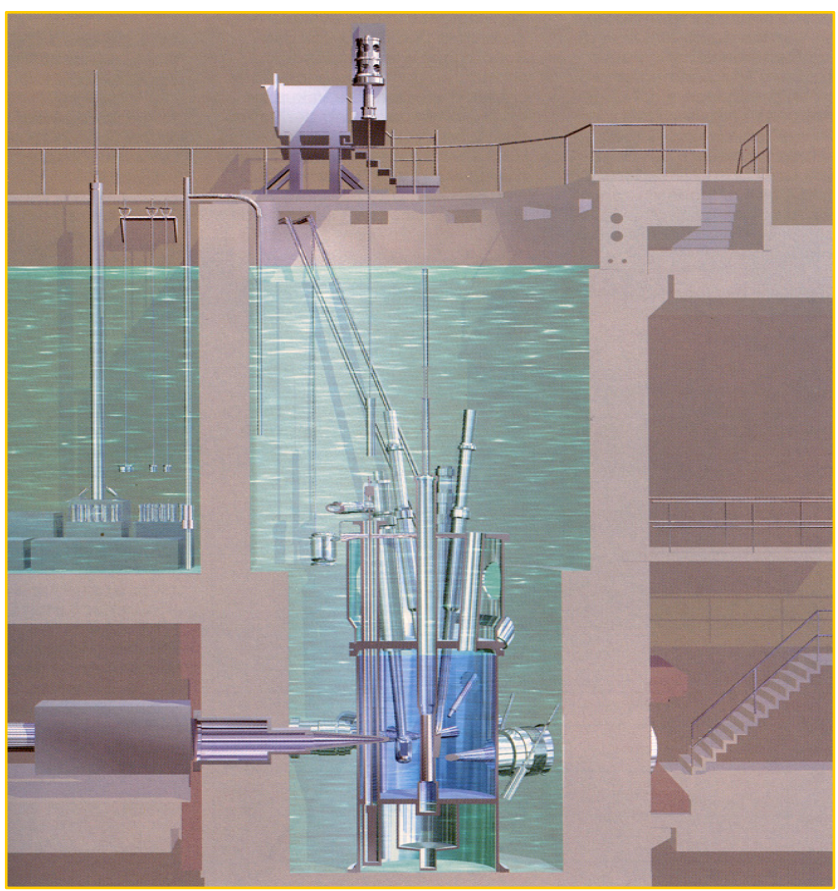

Figure 2: Conceptual design of the FRM II. The locations of various key components within the moderator tank are indicated

\subsection{Mechanical irradiation device}

For very short term irradiations requiring a thermal neutron fluence of not more than $5 \mathrm{E} 16 \mathrm{~cm}^{-2}$ the FRM II is equipped with a mechanical irradiation device. Before irradiation the sample is packed into an Al capsule which allows a maximum sample volume of $60 \mathrm{~cm}^{3}$ and is fixed to a nylon ribbon. The irradiation channel is a light water filled vertical thimble within the moderator tank which is accessible for the loading of irradiation samples through its open top. It is located in a distance of 1000 $\mathrm{mm}$ from the fuel element (center to center).

In order to load the $\mathrm{Al}$ capsule into the irradiation channel it is lowered into the irradiation channel by simply unwinding the Nylon ribbon from a coil (see figure 3).

The standard vertical irradiation position of the mechanical irradiation device is the center plane of the fuel element. The corresponding neutron flux densities were determined by means of activation of $\mathrm{Al}: \mathrm{Au}(1 \%)$ wires and $\mathrm{Ni}$ foils to be $\Phi_{\mathrm{th}}=1.1 \mathrm{E} 13$ $\mathrm{cm}^{-2} \mathrm{~s}^{-1}$ and $\Phi_{\mathrm{f}}=1.5 \mathrm{E} 10 \mathrm{~cm}^{-2} \mathrm{~s}^{-1}$. It is noteworthy that it is very easy to define additional irradiation positions with even lower neutron flux densities by choosing a higher vertical irradiation position within the irradiation channel. 
The mechanical irradiation device attracts a lot of interest from geological institutes from all over the world. Due to the comparatively low neutron flux density and its low gradient of only few percent over a typical sample length of $60 \mathrm{~mm}$ it offers an ideal experimental situation for the fission track dating technique of mainly apatite minerals. An overview of this technique can be found in (Gallagher 1998).

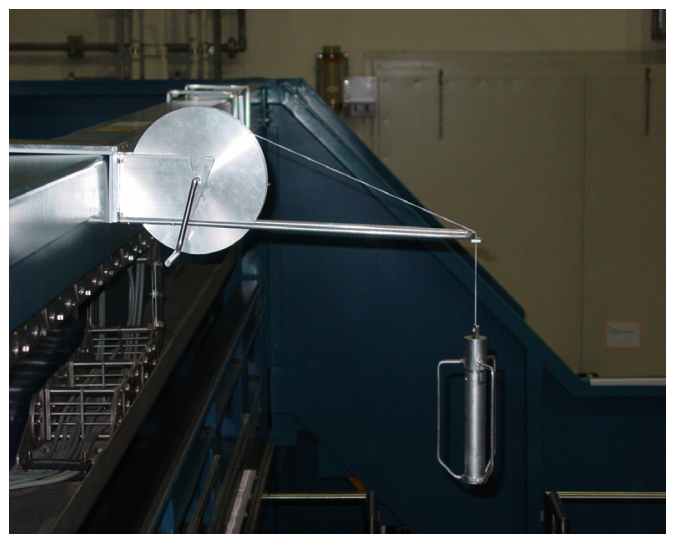

Figure 3: Mechanical irradiation device at the FRM II

\subsection{Rabbit irradiation facilities}

The FRM II is equipped with a conventional gas driven rabbit system which is mainly used for the activation of samples for neutron activation analysis and a water driven system which is suited for long term irradiations up to 14 days which means a thermal neutron fluence of above $1 \mathrm{E} 20 \mathrm{~cm}^{-2}$.

The gas driven system RPA (abbreviation of the German word Rohrpostanlage) offers in total 6 independent irradiation channels with different neutron flux density parameters. The differences are due to the fact that the depths of the irradiation channels within the moderator tank are graded. For all of these irradiation channels neutron flux densities and neutron temperatures were determined via the irradiation of flux monitors according to the Høgdahl or Westcott convention (Lin 2006). The results are shown in table 1.

The RPA uses carbon dioxide as process medium in order to avoid the undesired production of ${ }^{41} \mathrm{Ar}$ which would be generated during the irradiation of air. The irradiation capsules are made from polyethylene with an inner diameter of $\varnothing=15 \mathrm{~mm}$ and a length of $100 \mathrm{~mm}$. They are labelled individually as a precaution against mix-up and have a weight of approximately $10 \mathrm{~g}$. The maximum sample load is again $10 \mathrm{~g}$ but typically neutron activation samples weigh well below $1 \mathrm{~g}$.

After being loaded into the irradiation facility, the capsules are rinsed with fresh carbon dioxide again to avoid the undesired side irradiation of air, identified by the computer control and sent into the irradiation position. The stream of transport gas is generated by a ventilator only without the use of any pressure vessels; thus the pressure within the system is kept at only 1.2 bars abs. The transport velocity is about $10 \mathrm{~m} / \mathrm{s}$. The applicable neutron fluence is limited by the capsule material $\mathrm{PE}$ to $5 \mathrm{E} 17 \mathrm{~cm}^{-2}$. After completion of the irradiation the sample is blown out of the irradiation position and stopped in a well shielded decay position $(165 \mathrm{~mm} \mathrm{~Pb})$ where the induced gamma dose rate is measured. After the decay of the radioactivity below the limiting value of 6 $\mathrm{mSv} / \mathrm{h}$ in $50 \mathrm{~cm}$ (unshielded) the sample can be unloaded optionally in a shielded $(10 \mathrm{~cm})$ lead box which is equipped with manipulators for further handling or directly into a lead shielded transport container. Alternatively the sample can be automatically handed over to an independent pneumatic dispatch for direct transport into the neighbouring institute for radiochemistry of the Technische Universität München, one of the major customers for radioactive samples.

For longer term irradiation the water driven capsule irradiation facility KBA (abbreviation of the German word Kapselbestrahlungsanlage) is used. This system exhibits 2 independent irradiation channels each of which can be loaded by up to 5 irradiation capsules simultaneously. The central component of the KBA is a disc shaped storage device (so-called roundabout) being located approximately $4 \mathrm{~m}$ below the surface of the reactor's storage pool. This device is designed to manage up to 38 irradiation capsules (see figure 4).

For the long term irradiations in the KBA the samples are contained in standard capsules made from extremely pure $\mathrm{AlMg} 3$ which turned out to be necessary in order to allow unloading shortly after the irradiation which is possible due to the short halflives of the isotopes being generated from $\mathrm{Al}$ and $\mathrm{Mg}$. The available space within the capsule is a diameter of $\varnothing=26 \mathrm{~mm}$ and a length of $90 \mathrm{~mm}$, the maximum sample mass is $100 \mathrm{~g}$. Water sensitive samples are additionally sealed into tight quartz ampoules or welded Al-tubes before being placed into the irradiation capsule. The irradiation positions are located deep in the moderator tank. They are the most intense ones in FRM II. The corresponding neutron flux parameters (Lin 2006) are given in table 2 .

To launch an irradiation in the KBA the AlMg3 capsule is inserted into the loading tube. It sinks down into the "roundabout", which rotates the sample into a position which is connected to the irradiation position within the moderator tank. A pump generates a stream of pool water that transports the sample into the irradiation position. The speed of transport is only about $0.5 \mathrm{~m} / \mathrm{s}$ which is acceptable because of the long irradiation times. After completion of the irradiation the sample is brought back into the "roundabout" using the same way. It is kept in this position under water for the necessary decay time. For unloading from the KBA it is pumped in the first step into the position for dose rate measurement, which is still several meters below the surface of the pool. Only after verification of the conformity of the measured dose rate with the limiting value of $100 \mathrm{mSv} / \mathrm{h}$ (unshielded in a distance of $15 \mathrm{~cm}$ ) it is transported into a Pbshielded container that is waiting in the bottom position of a lift still $2.5 \mathrm{~m}$ below the pool surface. Otherwise it falls back into the "roundabout" for further decay. Optionally the sample can also be transferred into the hot cell of the FRM II.

The main application of the KBA up to now was the production of candidate isotopes for future radiopharmaceuticals e.g. ${ }^{177} \mathrm{Lu}$ or the commercial production of long lived isotopes like ${ }^{60} \mathrm{Co}$ for industrial applications. 
Table 1: Neutron flux density parameters for the pneumatic rabbit system RPA

\begin{tabular}{|c|c|c|c|c|c|}
\hline Irradiation position & $\Phi_{\text {th }}\left(\mathrm{cm}^{-2} \mathrm{~s}^{-1}\right)$ & $\Phi_{\text {epi }}\left(\mathrm{cm}^{-2} \mathrm{~s}^{-1}\right)$ & $\Phi_{\mathrm{f}}\left(\mathrm{cm}^{-2} \mathrm{~s}^{-1}\right)$ & $\mathrm{T}_{\mathrm{n}}\left({ }^{\circ} \mathrm{C}\right)$ & $\Phi_{\text {th }} / \Phi_{\mathrm{f}}$ \\
\hline & & & & & \\
\hline RPA-1 & $3.57 \mathrm{E} 13$ & $6.70 \mathrm{E} 09$ & $2.02 \mathrm{E} 09$ & 23.0 & 17673 \\
\hline RPA-2 & $1.52 \mathrm{E} 13$ & $3.18 \mathrm{E} 09$ & $4.11 \mathrm{E} 08$ & 30.3 & 36983 \\
\hline RPA-3 & $4.84 \mathrm{E} 12$ & $7.61 \mathrm{E} 08$ & $7.17 \mathrm{E} 07$ & 21.2 & 67503 \\
\hline RPA-4 & $7.26 \mathrm{E} 13$ & $2.34 \mathrm{E} 10$ & $5.56 \mathrm{E} 10$ & 23.8 & 1306 \\
\hline RPA-5 & $3.88 \mathrm{E} 13$ & $1.14 \mathrm{E} 10$ & $5.87 \mathrm{E} 09$ & 27.4 & 6610 \\
\hline RPA-6 & $7.12 \mathrm{E} 12$ & $1.24 \mathrm{E} 09$ & $1.51 \mathrm{E} 08$ & 27.6 & 47152 \\
\hline & \multicolumn{7}{|l}{} & & & \\
\hline
\end{tabular}

Table 2: Neutron flux parameters in the capsule irradiation facility

\begin{tabular}{|c|c|c|c|c|c|}
\hline Irradiation position & $\Phi_{\text {th }}\left(\mathrm{cm}^{-2} \mathrm{~s}^{-1}\right)$ & $\Phi_{\text {epi }}\left(\mathrm{cm}^{-2} \mathrm{~s}^{-1}\right)$ & $\Phi_{\mathrm{f}}\left(\mathrm{cm}^{-2} \mathrm{~s}^{-1}\right)$ & $\mathrm{T}_{\mathrm{n}}\left({ }^{\circ} \mathrm{C}\right)$ & $\Phi_{\text {th }} / \Phi_{\mathrm{f}}$ \\
\hline KBA 1-1 & & & & & \\
\hline KBA 1-2 & $1.29 \mathrm{E} 14$ & $2.82 \mathrm{E} 11$ & $3.87 \mathrm{E} 11$ & 11.7 & 333 \\
\hline KBA 2-1 & $9.35 \mathrm{E} 13$ & $1.07 \mathrm{E} 11$ & $1.94 \mathrm{E} 11$ & 8.9 & 483 \\
\hline KBA 2-2 & $1.07 \mathrm{E} 14$ & $8.18 \mathrm{E} 10$ & $2.06 \mathrm{E} 11$ & 11.2 & 520 \\
\hline
\end{tabular}

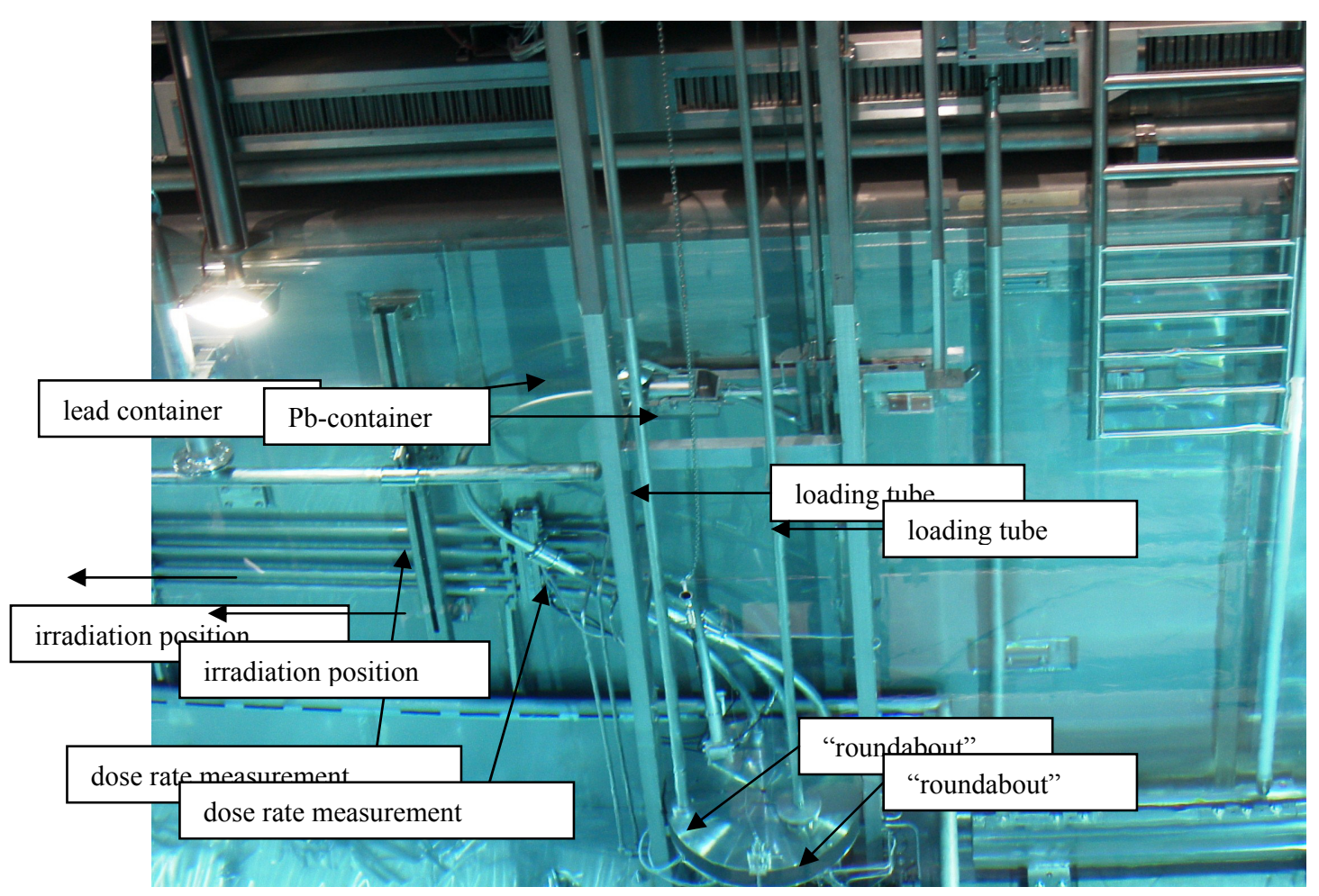

Figure 4: Capsule irradiation facility KBA of the FRM II

\subsection{Silicon doping facility}

The P-doping of Si by means of the neutron transmutation effect is based on the nuclear reaction

$$
{ }^{30} \mathrm{Si}(n, \gamma){ }^{31} \mathrm{Si} \stackrel{\beta^{-}}{\longrightarrow}{ }^{31} \mathrm{P}(1)
$$

The motivation for doping $\mathrm{Si}$ in a nuclear reactor is the high homogeneity of the doping profile which is hardly achievable with other doping techniques. Therefore NTD Silicon is mainly used in power consuming electronic components.

The basic condition to be met for a successful doping of Si in a research reactor is to provide an irradiation position with a very homogeneous neutron flux density profile. At FRM II additionally a design was required that allowed the irradiation of ingots with a diameter of up to $200 \mathrm{~mm}$ and a height of the stack of ingots to be irradiated simultaneously of $500 \mathrm{~mm}$. Like for the mechanical irradiation facility presented earlier the irradiation channel is a 
vertical, light water filled thimble in a distance of $1000 \mathrm{~mm}$ (center to center) from the reactor core.

Already during the commissioning phase of FRM II the vertical neutron flux density profile in the irradiation position was determined using a simplified irradiation rig to be manipulated via the crane of the rector hall. For this purpose two Si ingots $(\varnothing=$ $150 \mathrm{~mm}$ ) were equipped with a total of $15 \mathrm{Al}: \mathrm{Au}(2 \%)$ monitor wires and irradiated. In order to increase the radial homogeneity of doping the ingots were rotated during irradiation with a frequency of $5 \mathrm{~min}^{-1}$. The result was a maximum inhomogeneity of the flux profile of $12 \%$ along the cylinder axis, in contrast the radial inhomogeneity was below $3 \%$. On the basis of these data the profile of a Ni-absorber layer - the so-called liner - for the smoothening of the neutron flux density along the axis of the $\mathrm{Si}$ ingots was calculated using MCNP methods. For the final $\mathrm{Si}$ doping facility this absorber layer was embedded into the Al-tube surrounding the irradiation position by means of an injection moulding technique.

The absolute thermal neutron flux density in the Si ingot was determined to be $\Phi_{\text {th }}=1.7 \mathrm{E} 13 \mathrm{~cm}^{-2} \mathrm{~s}^{-1}$. the ratio of thermal and fast neutron flux density $\Phi_{\mathrm{th}} / \Phi_{\mathrm{f}} \approx 1700$. A very wishful consequence of this high ratio is that the irradiation does not produce many extended defect clusters which can hardly be annealed. Therefore the target resistivity for $\mathrm{Si}$ to be irradiated in FRM II can be as high as $1050 \Omega \mathrm{cm}$.
$150 \mathrm{~mm}$ with respect to the vertical position of the control rod in the fuel element. On the other hand the change of the form of the neutron flux density during the reactor cycle turned out to be of minor importance.

The semi-automatic final $\mathrm{Si}$ doping facility was taken into operation in January 2007. All major parameters like the transport route of the Si within the rector pool, the rotation frequency of the $\mathrm{Si}$ during irradiation, the irradiation time and many others are controlled by a computer system. After only 48 hours of decay time the $\mathrm{Si}$ is extracted from the pool and can be released from the regulations for radioactive material after cleaning in an ultrasonic bath.

In the first year of operation an amount of almost $4 \mathrm{t}$ of Si was irradiated at FRM II for various customers from Europe and Asia.

\subsection{Cancer treatment by irradiation with fast neutrons}

Already before FRM II there was an experience regarding the tumour therapy with fast neutrons at Technische Universität München from the treatment of more than 700 patients which had been carried out at the former FRM reactor between 1985 and 2000. Therefore it was decided to equip also FRM II with a medical irradiation device using fast neutrons. The patient irradiations at FRM II started in 2007 under the guidance and medical responsibility of Klinikum rechts der Isar which is also part of the Technische Universität München.
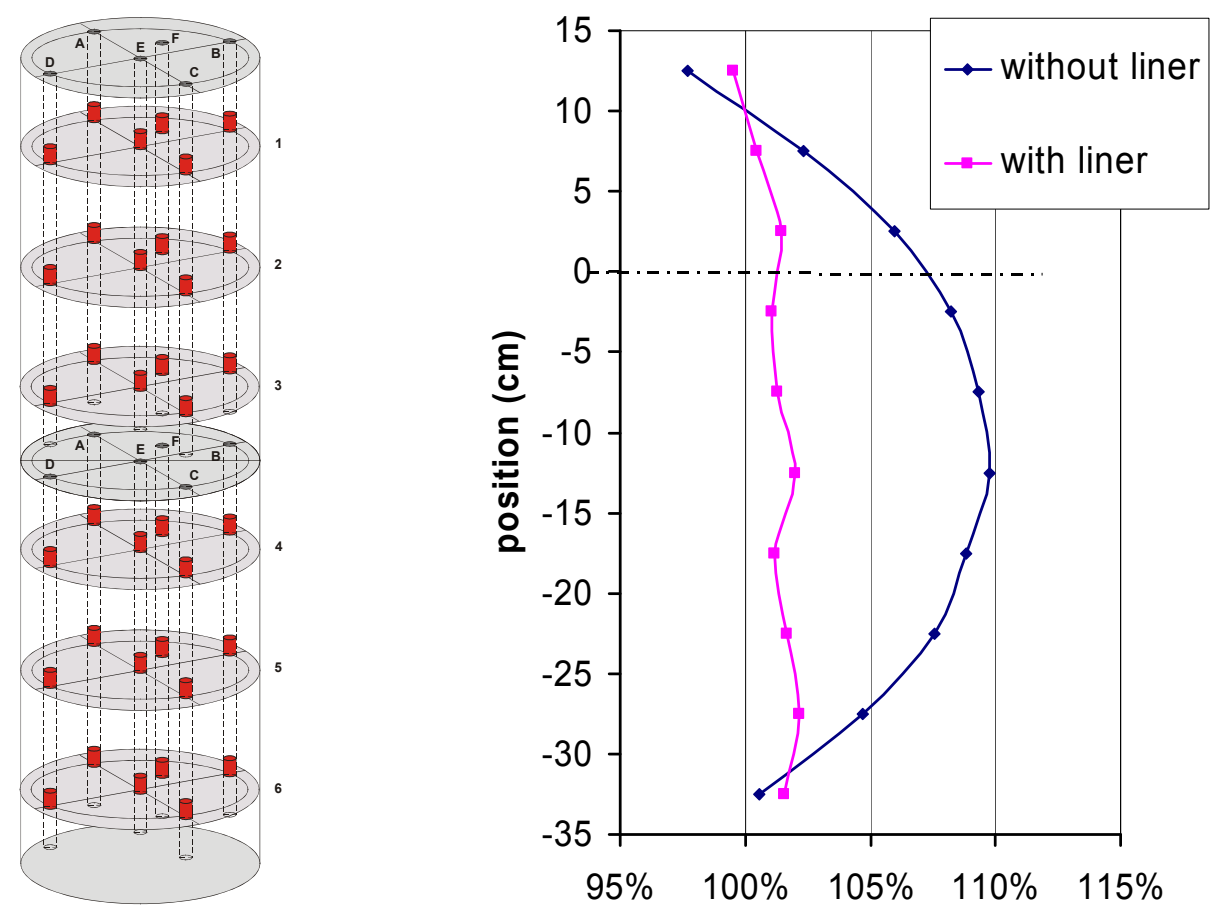

Figure 5: Si ingot equipped with flux monitors and measured neutron flux density profile with and without Ni-liner

In addition to the use of a Ni liner and the rotation of the Si during irradiation also the vertical shift of the neutron flux density with increasing burn-up of the fuel element has to be corrected for. For this purpose the entire irradiation facility can be lifted by up to
The biological advantage of using fast neutrons $(\mathrm{E} \approx 1 \mathrm{MeV})$ for the therapy of malignant tumours is their high radiobiological efficiency as compared to conventional projectiles. Due to their interaction pattern with human tissue it is most favourably applied 
to slowly growing and well differentiated tumours mainly of the neck and head. In addition all shallow tumour lesions like skin metastases from various cancer diseases as well as chest wall metastases of breast cancer are suited for neutron irradiation therapy particularly when pre-treated by irradiation projectiles with a low linear energy transfer.

The fast neutrons are generated in the so-called converter facility, the main component of which is a pair of fuel plates containing a total of $498 \mathrm{~g}{ }^{235} \mathrm{U}$. The converter plates are located at the edge of the moderator tank in close vicinity to the entrance window of a neutron beam tube. The fission neutrons from these fuel plates are hardly moderated since they have to penetrate only few $\mathrm{mm}$ of water and some thin Al-foils before entering into the beam tube. The beam tube itself contains four shutter drums. It ends at the outer surface of the biological shielding of FRM II in the heavily shielded room for patient treatment. In order to establish the effect of the fast neutrons the non wishful gamma dose rate is decreased by a $35 \mathrm{~mm} \mathrm{~Pb}$-filter. In addition a B4C filter suppresses thermal and epithermal neutrons. The beam is shaped by a multi leaf collimator that allows to match the area of neutron radiation to the contour of the tumour up to $20 * 30 \mathrm{~cm}^{2}$ in maximum.

The fast neutron flux density at the irradiation position is 7.1E8 $\mathrm{cm}^{-2} \mathrm{~s}^{-1}$; the mean neutron energy is $1.6 \mathrm{MeV}$. The dose distribution along the depth of a water phantom was determined experimentally in order to provide the basis for working out medical treatment plans. With the combination of filters for therapeutic irradiations $\left(10 \mathrm{~mm} \mathrm{~B}_{4} \mathrm{C}\right.$ and $\left.35 \mathrm{~mm} \mathrm{~Pb}\right)$ the ratio of neutron / photon decreases from 3.6 at the surface of the water phantom to 1.8 and a depth of $50 \mathrm{~mm}$. The drastic decrease of the dose rate with increasing depth is the reason that only superficial tumours are irradiated with fast neutrons.

\section{SUMMARY}

Since 2005 the Forschungsneutronenquelle Heinz Maier-Leibnitz (FRM II), Germany's youngest research reactor, is in operation at the Technische Universität München. Besides being used for the basic physical research by means of beam tube experiments it is equipped with various irradiation devices serving for a large variety of applications in research, medicine and industry. The demand for the irradiation service increased continuously in the 3 years of FRM II operation from about 150 irradiations in 2005 to more than 500 irradiations in 2007. Presently there is no need to expect an end of this trend, since a lot of new ideas and inquiries for additional irradiations are presented to FRM by internal and external interested parties.

\section{REFERENCES}

GALLAGHER K., BROWN R., JOHNSON C., (1998), Fission track analysis and its applications to geological problems, Annu. Re. Earth Planet Sci., 26, 519-572

LIN X.; HENKELMANN R., TÜRLER A., GERSTENBERG H., DE CORTE F., (2006), Neutron flux parameters at irradiation positions in the new research reactor FRM-II, Nuclear instruments and methods in Physics Research A, 564 641-644

SCHRECKENBACH K., GERSTENBERG, H., (2005), Status Report on the Nuclear Start-Up of FRM-II, Transactions, $9^{\text {th }}$ Int. Topical Meeting on Research Reactor Fuel Management, April 10-13, 2005, Budapest, Hungary, 114-118 